\title{
Helicobacter pylori in vivo causes structural changes in the adherent gastric mucus layer but barrier thickness is not compromised
}

\author{
J L Newton, N Jordan, L Oliver, V Strugala, J Pearson, O F W James, A Allen
}

\begin{abstract}
Background-It has been proposed that a pathogenic effect of Helicobacter pylori is a weakening of the protective mucus barrier; however, this remains controversial.

Aims-To clarify the effects of $H$ pylori infection on the mucus gel barrier in vivo. Methods-Mucus gel polymeric structure and the thickness of the adherent mucus barrier were measured in endoscopic biopsy samples in subjects with and without $H$ pylori infection.

Results-There was a significant $18 \%$ reduction in the proportion of polymeric gel forming mucin in the adherent mucus layer in $H$ pylori positive compared with negative subjects. There was no change in the adherent mucus thickness between $H$ pylori positive and negative subjects without gastric atrophy (mean (SD): 104 (26) $\mu \mathrm{m}, 106$ (30) $\mu \mathrm{m}$ respectively). There was however a significant reduction in mucus thickness in those $H$ pylori positive subjects with underlying gastric atrophy (84 (13) $\mu \mathrm{m}, p=0.03$ ) compared with those without atrophy.

Conclusions-A partial breakdown in gel forming structure of the gastric mucus barrier does occur in $H$ pylori infection per se but this is insufficient to cause a collapse of the mucus barrier.

(Gut 1998;43:470-475)
\end{abstract}

Keywords: Helicobacter pylori; gastric mucus

Department of Physiological Sciences J L Newton

N Jordan

L Oliver

V Strugala

J Pearson

A Allen

Department of Medicine, University of Newcastle-upon-

Tyne, UK

O F W James

Correspondence to: Dr J Newton, Department of Medicine, The Medical

School, Framlington Place,

University of

Newcastle-upon-Tyne,

Newcastle-upon-Tyne

NE2 4HH, UK.

Accepted for publication 4 March 1998

and is an aetion is an aetiological factor in the pathogenesis of peptic ulceration. ${ }^{1} \mathrm{H}$ pylori is located within the adherent mucus barrier, particularly in the region of the gastric pit, close to the mucosal surface. ${ }^{2}$ The mucus barrier, together with epithelial bicarbonate secretion, is considered to be the first line of mucosal defence against acid and pepsin. ${ }^{3}$ This mucus layer also provides a protective environment for $H$ pylori to colonise. ${ }^{4}$ However, it is controversial whether or not $H$ pylori colonisation results in changes in mucus structure and production, thereby impairing its protective efficacy.

A weakening of the mucus barrier by $H$ pylori, leading in some cases to its collapse, has been proposed, based on the demonstration in vitro of a mucolytic proteinase in filtrates from cultures of $H$ pylori. ${ }^{5}{ }^{6}$ However, other groups have failed to show significant amounts of proteinase activity in either culture filtrates or extracts of $H$ pylori..$^{7-9}$ A non-proteolytic, urea dependent breakdown of the mucus gel by filtrates of $H$ pylori has been observed and explained by urease activity producing ammonium ions which disrupted mucus lipid interactions. ${ }^{8}$ A study in vivo looking at mucus gel in gastric washouts, but not from the adherent mucus gel barrier, showed that mucus viscosity from $H$ pylori infected subjects was not reduced compared with that from noninfected subjects. ${ }^{10}$ One study in vivo, observing unfixed sections of gastric mucosal biopsy specimens, showed an up to $50 \%$ reduction in mean mucus thickness of the adherent mucus gel barrier in patients with $H$ pylori infection, compared with non-infected controls. ${ }^{11} \mathrm{Re}-$ cently decreased expression of MUC5AC and aberrant expression of the glandular mucin MUC6 in gastric epithelial cells has been shown to occur with $H$ pylori infection. ${ }^{12}$

In the present study we set out to clarify the effects of $H$ pylori infection on the mucus gel barrier in vivo. We have assessed the relative amounts of gel forming polymeric mucin and the lower sized non gel-forming mucin in endoscopic biopsy specimens and mucosal brushings. Detailed rheological studies in vitro have shown that a direct relation exists between the percentage of the total mucin sample that is in the polymeric form and the strength and quality of the gel. ${ }^{13}$ We have also reassessed changes in gastric adherent mucus thickness in $H$ pylori infected subjects without pathology. This involved staining cryostat sections of endoscopic biopsy specimens with a modified periodic acid-Schiff's/Alcian blue method. ${ }^{14}$ Studies in the rat model show that this method preserves the mucus gel barrier intact without significant disruption, giving values close to those observed in vivo.

\section{Methods}

SUBJECTS

All subjects were attending for routine diagnostic gastroscopy for upper abdominal discomfort: none had a past history of peptic ulceration or other known gastric pathology. All were shown to have a macroscopically normal stomach. None was taking non-steroidal anti-inflammatory medication, or acid suppressive therapy. All subjects had fasted for a minimum of 12 hours.

\section{DETERMINATION OF H PYLORI STATUS}

$H$ pylori status was determined for each subject by both serology and histology.

Serological diagnosis involved the detection of IgG antibodies using the Helisal kit (Cortecs 
Diagnostics Ltd, UK). Four endoscopically obtained gastric biopsy specimens from each patient (two antral, two body), stained using haematoxylin and eosin and modified Giemsa, were assessed for the presence or absence of $H$ pylori and gastric atrophy. A subject was only considered to be infected with $H$ pylori if both serology and histology were positive, and a subject non-infected only if both were negative. Subjects were excluded if there was ambiguity between test results. Two sets of patients were recruited:

Group 1-measurement of adherent mucus thickness-Endoscopic biopsy specimens from $20 \mathrm{H}$ pylori negative subjects and 20 age matched $H$ pylori positive subjects were taken. Nine of the $H$ pylori positive group had histological evidence of gastric atrophy; none of the $H$ pylori negative group had atrophy. No subjects had intrinsic factor antibodies.

Group 2-determination of polymeric mucin content in the total mucin sample-Endoscopic biopsy specimens and mucosal brushings were taken from $24 \mathrm{H}$ pylori negative subjects and 24 age matched $H$ pylori positive subjects. Seven of the $H$ pylori positive subjects had gastric atrophy; none of the $H$ pylori negative subjects had evidence of gastric atrophy. No subjects had intrinsic factor antibodies.

MEASUREMENT OF GASTRIC ANTRAL MUCUS

THICKNESS IN BIOPSY SAMPLES

Four standard endoscopic biopsy specimens, all from within $2 \mathrm{~cm}$ of the pylorus, were wrapped in a $1 \mathrm{~cm}^{2}$ cube of pig liver to act as a support medium, and then immediately snap frozen with liquid nitrogen and stored at $-20^{\circ} \mathrm{C}$.

Cryostat sections were processed and stained using a modified periodic acid-Schiff's/Alcian blue stain (PAS/AB) according to the method of Jordan et al. ${ }^{14}$ This method, unlike previous histological techniques, reduces the use of dehydrating steps that shrink and remove the adherent mucus gel (harsh fixation, prolonged use of organic solvents, and use of resinous mountants).

Sections were pretreated in $100 \%$ ethanol for 10 minutes, rinsed in water, then immersed in $3 \%$ acetic acid for two minutes. Sections were stained for 2.5 hours in $1 \%$ Alcian blue $(\mathrm{pH}$ $2.5)$, rinsed in running tap water, then oxidised for 10 minutes in $1 \%$ periodic acid at room temperature. After washing in running tap water, sections were immersed in Schiff's reagent for 15 minutes. Three changes of $0.5 \%$ sodium metabisulphite were followed by a further rinse in tap water. Sections were fixed in paraformaldehyde vapour and mounted in gelatin.

Sixty measurements of mucus thickness (10 from each of six sections) were made from each individual using an eye piece graticule at intervals of approximately $250 \mu \mathrm{m}$ along the gastric mucosal surface, by an operator unaware of the $H$ pylori status. In the rat animal model this method gives mucus thickness values of a mean (SD) of 176 (10) $\mu \mathrm{m}$ for whole rat mucosa and 183 (25) $\mu \mathrm{m}$ for biopsied rat mucosa when processed using the above method. This showed that the taking of biopsy specimens and wrapping them in the liver support does not distort the mucus gel layer.

DETERMINATION OF THE POLYMERIC MUCIN CONTENT IN ENDOSCOPIC BIOPSY SAMPLES Microgram quantities of mucin were fractionated according to size by sodium dodecyl sulphate polyacrylamide gel electrophoresis (SDS-PAGE) using the method of Rankin et $a l .{ }^{15}$ In this method the large molecular weight gel forming polymeric mucin remains in the stacking gel while the smaller molecular sized degraded mucin enters the running gel.

For SDS-PAGE, purified mucin samples were dissolved in a non-reducing $\mathrm{pH} \quad 6.8$ buffer, $12.5 \mathrm{mM}$ Tris, $0.4 \%$ (wt/vol) sodium dodecyl sulphate, $2 \%$ (vol/vol) glycerol, $0.0002 \%$ bromophenol blue. Samples were boiled for two minutes, then fractionated on $4-15 \%$ gels (stacking gel 3\%) for $360 \mathrm{vH}$ followed by staining for mucin with PAS. ${ }^{16}$ Gels were scanned using a densitometer at $555 \mathrm{~nm}$ for quantitation of PAS positive bands.

Initial experiments were performed to validate the electrophoretic method for human gastric mucin purified from endoscopic biopsy specimens and brushings. In order to prepare sufficient gastric mucus for these validation studies human gastric mucin was isolated from pooled samples of gastric juice aspirated from nasogastric tubes placed in normal volunteers. Gastric juice was filtered through glass wool which retained the mucus gel. These glass wool plugs and mucus gel were homogenised in 1/15 $\mathrm{M}, \mathrm{pH} 6.5$ phosphate buffer, with a cocktail of protease inhibitors $(1 \mathrm{mM}$ iodoacetamide, 100 $\mathrm{mM}$ aminocaproic acid, $5 \mathrm{mM}$ benzamidine hydrochloride, $10 \mathrm{mM}$ EDTA, $10 \mathrm{mM}$ N-ethylmaleimide, and $1 \mathrm{mM}$ phenyl methyl sulphonyl fluoride). ${ }^{17}$ The mucin in the supernatant was fractionated using equilibrium density gradient centrifugation in caesium chloride (starting density $1.42 \mathrm{~g} / \mathrm{ml}$ ). ${ }^{18}$ The resultant density gradient was separated into nine equal fractions and assayed for glycoprotein using the PAS reaction. ${ }^{19}$ Mucin rich fractions were pooled, dialysed exhaustively against distilled water, and freeze dried.

This human gastric mucin derived from gastric juice was fractionated using Sepharose $2 \mathrm{~B}-\mathrm{Cl}$ column chromatography $(30 \times 1.5 \mathrm{~cm})$. The sample was dissolved in $0.5 \mathrm{ml}$ of $0.2 \mathrm{M}$ sodium chloride containing $0.02 \%$ sodium azide and eluted downwards. Sixty fractions of $1 \mathrm{ml}$ were collected and glycoprotein measured by the PAS reaction. ${ }^{19}$ Two PAS positive peaks eluted from column, one which comprised 45 (5) $\%(n=3)$ of the total PAS positive material and excluded from Sepharose 2B; this was equivalent to large molecular weight gel forming polymeric mucin. The remaining PAS positive material was included and eluted in a similar position to lower molecular weight proteolytically degraded human gastric mucin. ${ }^{18}$

Fractionation of this sample of human gastric mucin from gastric juice on SDSPAGE was carried out. When scanned, two bands of PAS positive material were present. Band "a" was a broad band at the point of 

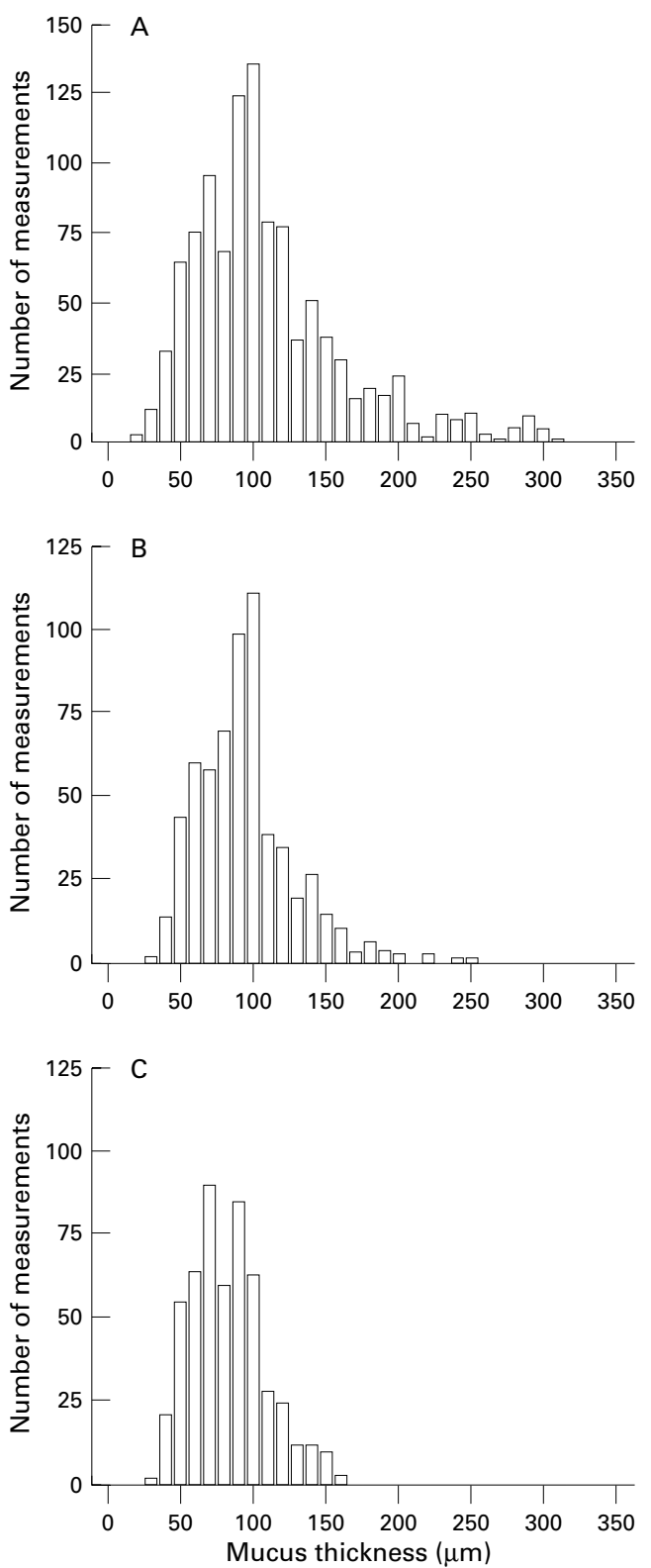

Figure 1 Individual mucus thickness measurements (measurements within $\pm 5 \mu \mathrm{m}$ ) shown according to H pylori status and the presence or absence of underlying gastric atrophy. (A) H pylori negative; (B) H pylori positive without atrophy; (C) H pylori positive with atrophy.

sample application in the stacking gel, corresponding to large sized gel forming polymeric mucin equivalent to material excluded from the Sepharose 2B column. Band "b", which entered the running gel, was clearly separated from band "a" and corresponded to standard lower molecular weight proteolytically degraded mucin which was included on Sepharose $2 \mathrm{~B}$. The amounts of PAS positive material

Table 1 Gastric antral adherent mucus thickness

\begin{tabular}{llll}
\hline Subjects & Number & Mean age $(y)$ & Mucus thickness $(\mu m)$ \\
\hline H pylori negative & 20 & 57.4 & $106(30)$ \\
H pylori positive total & 20 & 59.1 & $94(24)$ \\
H pylori positive without atrophy & 11 & 56.6 & $104(26)$ \\
H pylori positive with atrophy & 9 & 64.8 & $84(13)^{\star}$
\end{tabular}

${ }^{\star}$ Mean (SD) mucus thickness is significantly lower in $H$ pylori positive subjects with gastric atrophy compared with those without $(\mathrm{p}=0.03)$. in the two peaks, excluding band "a" and including band " $\mathrm{b}$ ", were calculated by scanning the gel at $555 \mathrm{~nm}$ and measuring the area under the peaks. Estimation of the amount of PAS positive material in band " $a$ " following gel electrophoresis of standard mucin samples was a mean of $45 \% \quad(n=3)$ large sized polymeric mucin. Gel filtration on Sepharose 2B of the same mucin samples gave a mean of $43 \%$ large sized polymeric mucin. The values for fractionation of individual samples by the two different methods were always within $3 \%$ of each other.

Collection of samples for mucin analysis was restricted to four biopsy specimens taken from each individual, which involved microgram quantities that were too small for routine glycoprotein assays on caesium chloride fractions. Therefore mucin containing fractions following density gradient centrifugation were pooled according to density $(1.41-1.5 \mathrm{~g} / \mathrm{ml})$. The correct density was determined using mucin from gastric juice (as described above), and also three separate fractionations of 12 pooled human gastric antral biopsy specimens (four from each of three patients) using the methodology described below for individual patient samples. Individual fractions from equilibrium density centrifugation of these mucin samples were assayed for density and for mucin by the PAS method. ${ }^{19}$

From each subject the following samples were obtained: four standard endoscopic biopsy specimens from within $2 \mathrm{~cm}$ of the pylorus which were pooled and homogenised; and a surface mucus sample obtained by passing a $3 \mathrm{~mm}$ bristle standard endoscopic cytology brush four times over the pyloric antrum.

Human samples were each separately collected into $10 \mathrm{ml}$ of inhibitor buffer. ${ }^{17}$ Samples were then each fractionated separately in a caesium chloride density gradient. ${ }^{18}$ Subsequently mucin rich fractions from the biopsy specimens and brushings with density $1.41-1.5 \mathrm{~g} / \mathrm{ml}$ were separately pooled, dialysed exhaustively against distilled water, and then freeze dried. Repeated electrophoretic fractionation by SDS-PAGE of the same sample gave reproducible results with the same percentage ratio (within $3 \%, n=3$ ) of larger sized polymeric mucin to smaller sized degraded mucin.

Ethical permission for this study was granted by the South of Tyne Health Commision Ethical Committee, Queen Elizabeth Hospital, Gateshead. All subjects gave written informed consent.

\section{STATISTICAL ANALYSIS}

Results are expressed as means (SD). Student's unpaired $t$ test was used to test for significance between groups. Values with $\mathrm{p}<0.05$ were taken as significant.

\section{Results}

GASTRIC ADHERENT MUCUS THICKNESS

In all subjects, following staining with the modified PAS/AB stain the adherent mucus gel layer appeared as a thick, continuous pink, staining layer, covering the antral mucosal 


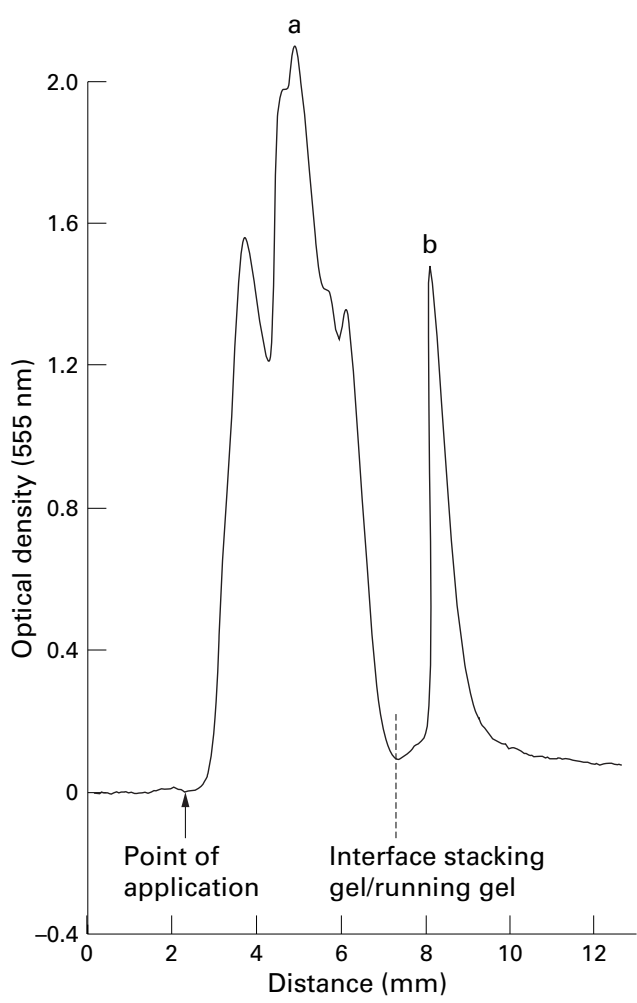

Figure 2 Representative SDS-PAGE of gastric antral mucin showing 2 distinct bands. Band a: polymeric large molecular weight mucin that enters the stacking gel; mucin applied to gel enters the stacking gel and diffuses around the point of application as one broad band. Band b: smaller sized mucin that enters the running gel; dotted line shows the interface between the stacking and the running gel.

surface. The mean mucus thickness of this layer in $H$ pylori negative subjects was 106 (30) $\mu \mathrm{m}$; figure 1 shows the distribution of individual readings.

There was no significant difference in mucus thickness measurement between $H$ pylori negative subjects and those infected with $H$ pylori without gastric atrophy (mean 104 (26) $\mu \mathrm{m}$; $\mathrm{n}=11)$. In contrast, subjects who were $H$ pylori positive with gastric atrophy $(n=9)$ had a significant decrease $(p=0.03)$ in mean mucus thickness measurement (84 (13) $\mu \mathrm{m}$; table 1) when compared with both $H$ pylori negative subjects and $H$ pylori positive subjects without atrophy.

Analysis of individual mucus thickness measurements indicated that the significant reduction in mucus thickness in those with $H$ pylori associated gastric atrophy was reflected in fewer higher thickness measurements compared with the other two groups (fig 1). However, a minimum thickness of the mucus layer was always maintained and was never less than $20 \mu \mathrm{m}$. Over $95 \%$ of individual thickness readings were at least $50 \mu \mathrm{m}$ in all three groups.

DETERMINATION OF POLYMERIC MUCIN CONTENT For all subjects gel electrophoresis of samples of the adherent mucus gel gave two PAS staining bands: the excluded PAS positive band "a" extending from the origin into the stacking gel; and an included PAS positive band " $b$ " which had entered the running gel and clearly separated from band "a" (fig 2).

The optical density of the two bands at 555 $\mathrm{nm}$ was measured and the ratio of area under the two peaks calculated to give the percentage of mucin that was polymeric.

Mucin from brushings (primarily adherent surface gel) taken from $H$ pylori negative subjects had a mean percentage of polymeric mucin of $72(12) \%$ (table 2). The mean percentage of polymeric mucin in subjects infected with $H$ pylori was 59.5 (16)\%. This represented a significant $18 \%$ reduction $(\mathrm{p}<0.02)$ in the proportion of mucin that was polymeric in the brushings from $H$ pylori infected subjects. Splitting the total $H$ pylori positive group into those with and those without gastric atrophy, gave mean percentages of polymeric mucin in mucus brushings of 60 (13)\% and 59 (17)\% respectively; these values were not significantly different from each other although both were significantly lower than the value for $H$ pylori negative subjects.

Mucin from biopsy samples, representing both the adherent mucus gel layer and presecreted intracellular mucus, gave the same electrophoretic pattern as that seen with brushings. The mean percentage of large molecular weight gel forming polymeric mucin to degraded mucin in the biopsy samples from $H$ pylori negative subjects was 80.5 (9)\%. The mean percentage of polymeric mucin in subjects infected with $H$ pylori was 76 (13)\% which, while lower than that in uninfected subjects, was not significantly so (table 2 ; fig 3 ). There was no significant difference in the percentage ratio of polymeric mucin content in the biopsy samples between those with and those without $H$ pylori associated gastric atrophy.

The amounts of polymeric mucin in biopsy specimens from $H$ pylori negative and positive subjects were significantly higher $(7.5 \%$ and $16.5 \%$ respectively) than those in brushings for all subject groups $(p=0.02$; see table 2$)$.

Table 2 Percentage polymeric gel forming mucin content in biopsy specimens and brushings of $H$ pylori positive and negative age matched subjects

\begin{tabular}{|c|c|c|c|c|c|c|}
\hline \multirow[b]{3}{*}{ Subjects } & \multirow[b]{3}{*}{$n$} & \multirow[b]{3}{*}{ Mean age $(y)$} & \multicolumn{4}{|c|}{ Polymeric mucin content in total mucin sample (\%) } \\
\hline & & & \multicolumn{2}{|l|}{ Biopsy $(A)$} & \multicolumn{2}{|c|}{ Mucosal brushing (B) } \\
\hline & & & Mean (SD) & Range & Mean $(S D)$ & Range \\
\hline H pylori negative & 24 & 62 & $80.5(9) \dagger$ & $60-100$ & $72(12)$ & $50-97$ \\
\hline H pylori positive total & 24 & 61.5 & $76(13) \dagger$ & $49-96$ & $59.5(16)^{\star}$ & $34-87$ \\
\hline$H$ pylori positive without atrophy & 17 & 57.5 & $74(14)$ & $49-96$ & $59(17)$ & $34-87$ \\
\hline H pylori positive with atrophy & 7 & 67.5 & $83(8)$ & $72-92$ & $60(13)$ & $44-82$ \\
\hline
\end{tabular}

$\star$ Percentage polymeric mucin content in brushings was significantly lower in $H$ pylori positive compared with negative subjects.

†Percentage polymeric mucin content in biopsy samples was significantly higher $(\mathrm{p} \leq 0.02)$ than in the brushings in both those with and without $H$ pylori infection. 

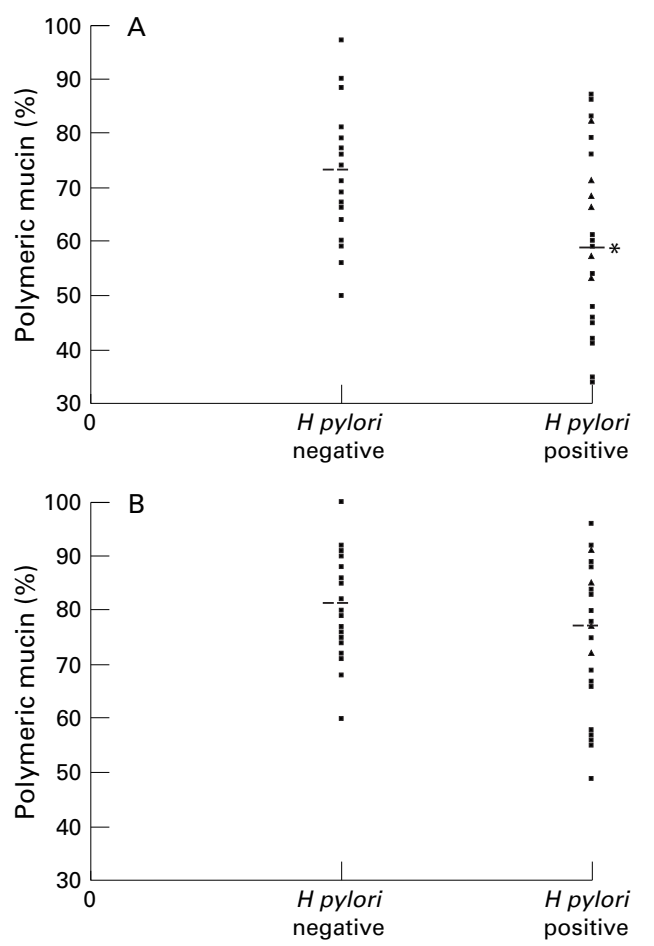

Figure 3 Percentage ratio of large molecular weight polymeric mucin in: (A) mucosal surface brushings (adherent mucus gel layer); and (B) biopsy samples (intracellular mucus and adherent mucus gel layer). Dotted lines represent mean values. ${ }^{*} p=0.01$.

\section{Discussion}

The gel strength and thickness of the adherent mucus gel layer are key parameters in assessing the protective efficacy of the gastric mucus barrier. ${ }^{3}$ In the present study we have shown that $H$ pylori infection is associated with a breakdown in mucus structure in vivo. This is reflected by an $18 \%$ decrease in the percentage of mucin that is polymeric in mucosal brushings, representative of the adherent gastric mucus. However this decrease in gel forming polymeric mucin was not matched by a corresponding decrease in the mean thickness of the adherent mucus gel layer except in $H$ pylori positive individuals who had developed gastric atrophy. These data indicate that while $H$ pylori does cause a significant reduction in gel strength, the mucus barrier remains intact.

The relevance to the mucus barrier of the reduction in polymeric mucin content with $H$ pylori infection can be seen from rheological studies which show a linear correlation between gel strength and the percentage of mucin in the polymeric form..$^{13}$ These previous in vitro studies showed that the polymeric mucin content must fall below $30 \%$ before the mucus assumes the properties of a viscous liquid rather than that of a gel. The mean value of $72 \%$ for polymeric mucin content in mucosal brushings from uninfected subjects is indicative of a firm viscoelastic mucus gel. The mean value of $59 \%$ polymeric mucin for the brushings from $H$ pylori infected subjects does indicate a weaker gel structure. However, all the values for individual subjects obtained in the present study are above that where there is a collapse of structure and the mucus no longer exists as a gel. Interestingly, earlier studies ${ }^{20}$ on mucus scrapings from resected normal gastric mucosa (Whipple's resection for carcinoma of the head of pancreas) gave a polymeric mucin content of $69 \%$, a value close to that found for the brushings in this study. Also in this earlier study a mean polymeric mucin content of only $34 \%$ was found from mucus scraped from resected mucosa of gastric ulcer patients, indicating a large scale collapse of the mucus gel barrier in this chronic condition.

The polymeric mucin content in biopsy specimens from $H$ pylori infected individuals was not significantly reduced compared with that in uninfected individuals. This can be explained by the specimens containing substantial stores of intracellular mucin which largely mask the reduction in the polymeric mucin content induced by $H$ pylori in the extracellular mucus gel. This supports the hypothesis that the observed partial breakdown in the extracellular mucus is caused by $H$ pylori affecting its immediate mucus gel environment, rather than an effect on mucosal mucin biosynthesis.

It should be pointed out that the method used in this study, of firm brushing of the mucosal surface, while removing most of the surface mucus, may also remove some mucus cells and hence some intracellular mucus. Therefore if the local breakdown in mucin structure is associated with only the secreted mucus gel, the value of $18 \%$ reduction in its polymeric mucin content is likely to be a minimum value. This is reinforced by the close association of $H$ pylori with the apical cell membranes and tight junctions. ${ }^{2}$

The thickness of the mucus barrier and particularly its continuity is the other factor that will impact on its effectiveness. Our study has shown that $H$ pylori infection per se does not decrease the thickness of the mucus barrier. However, in individuals with $H$ pylori associated gastric atrophy, a small, but significant, decrease in mucus thickness was observed. An explanation for this may be the reduction in mucus secreting cells seen in association with $H$ pylori associated gastric atrophy. ${ }^{21}$

These results showing no change in mucus thickness associated with $H$ pylori infection alone are in keeping with a lack of effect of the organism on mucus secretion in vivo. In contrast, one previous study has shown as much as a $50 \%$ decrease in mucus thickness in $\mathrm{H}$ pylori infected individuals. ${ }^{11}$ However this study used the less precise technique of unfixed sections, ${ }^{22}$ which we find is not reliable on mucosal biopsy specimens. Furthermore, validation experiments in the rat show that the modified PAS/AB staining used in the present study ${ }^{14}$ gives thickness values twice that for unfixed sections, ${ }^{22}$ six times that for other histological methods, ${ }^{23}$ and compatible with those observed in vivo. ${ }^{24}$ In addition to these methodological problems the possible confounding effects of the presence of $H$ pylori associated gastric atrophy were not considered in the earlier study. ${ }^{11}$ This could explain why these authors observed a substantial decrease in adherent mucus thickness not seen in our studies. 
The mechanism by which $H$ pylori leads to a partial breakdown in the polymeric gel forming mucin structure in vivo is unclear. An $H$ pylori protease would be a plausible explanation; however, three different laboratories ${ }^{7-9}$ have now published studies failing to confirm the original observation of a substantial extracellular $H$ pylori protease activity. ${ }^{5}$ It is possible that neutrophil proteases associated with the inflammatory response to $H$ pylori might contribute to this partial breakdown of the mucin structure. More recent studies in vitro suggested that the mucus breakdown associated with $H$ pylori infection may be a function of the relatively alkaline conditions at the mucosal surface resulting from its high urease activity. ${ }^{8}{ }^{9}$

The observed $18 \%$ reduction in polymeric mucin structure in the adherent gel layer could in vivo be reflected throughout the adherent gel or represent local pockets of complete collapse of gel structure around the organism. The fact that there is no reduction in mucus thickness with infection would be compatible with $H$ pylori affecting its immediate gel environment near the mucosal surface and causing such local collapse. This would facilitate the movement of $H$ pylori through the mucus gel in its immediate vicinity while at the same time the organism would be preserving its overall protective environment within the mucus gel.

A recent report ${ }^{25}$ observing less dense adherent mucus in the area around $H$ pylori would support this hypothesis of a local collapse of gel structure around the organisms. Furthermore, a structurally intact gel away from these pockets of collapsed gel would explain the observed absence of any change in viscoelastic properties of sloughed off mucus gel in washouts from $H$ pylori infected subjects compared with those from uninfected subjects. ${ }^{10}$ Mucus from washouts does not however necessarily correlate with the state of the intact adherent gel. ${ }^{26}$

The general conclusion from this study is that $H$ pylori infection per se, does partially break down the mucin gel structure but without compromising the overall protective mucus barrier. Indeed $H$ pylori may have developed a mechanism which allows motility through the mucus gel while maintaining the full benefits of the protective environment in which it lives. A substantial breakdown of the mucus barrier appears to occur only when gastric atrophy or peptic ulceration ultimately supervenes.

J Newton was supported by a grant from the Astra Foundation. N Jordan was supported by a grant from the BBSRC.
1 Marshall BJ. Helicobacter pylori. Am $\mathcal{f}$ Gastroenterol 1994;89:S116-28.

2 Thomsen LL, Gavin JB, Tasman-Jones C. Relation of Helicobacter pylori to the human gastric mucosa in chronic gastritis of the antrum. Gut 1990;31:1230-6.

3 Allen A, Flemstrom G, Garner A, et al. Gastroduodenal mucosal protection. Physiol Rev 1993;73:823-47.

4 Marshall BJ, Barrett LJ, Prakash C, et al. Urea protects Helicobacter (Campylobacter) pylori from the bactericidal effect of acid. Gastroenterology 1990;99:696-702.

5 Slomiany BL, Bilski J, Sarosiek J, et al. Campylobacter pylori degrades mucin and undermines gastric mucosal integrity. Biochem Biophys Res Commun 1987;144:307-14.

6 Sarosiek J, Slomiany A, Slomiany BL. Evidence for weakening of gastric mucus integrity by Campylobacter pylori. Scand f Gastroenterol 1988;23:585-90.

7 Baxter A, Campbell CJ, Cox DM, et al. Proteolytic activities of human Campylobacter pylori and ferret gastric of human Campylobacter pylori and ferret gastric mun 1989;163:1-7.

8 Sidebotham RL, Batten JJ, Karim QN, et al. Breakdown of gastric mucus in presence of Helicobacter pylori. $\mathcal{f}$ Clin Pathol 1991;44:52-7.

9 Oliver L, Newton J, Dettmar PW, et al. Effects of Helicobacter pylori colonisation on the adherent gastric mucus barrier. Immunology 1996;89:94.

10 Markesich DC, Anand BS, Lew GM, et al. Helicobacter pylori infection does not reduce the viscosity of human gastric mucus gel. Gut 1995;36:327-9.

11 Sarsosiek J, Marshall BJ, Peura DA, et al. Gastroduodenal mucus gel thickness in patients with Helicobacter pylori: a method for assessment of biopsy specimens. $A m \mathcal{F}$ Gastroenterol 1991;86:729-34.

12 Byrd JC, Yan P, Sternberg L, et al. Aberrant expression of gland-type gastric mucin in the surface epithelium of Helicobacter pylori infected patients. Gastroenterology 1997;113:455-64.

13 Sellers LA, Allen A, Morris ER, et al. Mucus glycoprotein gels: role of glycoprotein polymeric structure and carbohygels: role of glycoprotein polymeric structure and carbohy-
drate side chains in gel formation. Carbohydr Res 1988;178: 93-110.

14 Jordan N, Newton JL, Pearson JP, et al. A new histological staining technique shows a continuous adherent mucus layer in rat and human stomach. Phys Soc 1996;77P:C47 .

15 Rankin BJ, Srivastava ED, Record CO, et al. Patients with ulcerative colitis have reduced mucin polymer content in the adherent colonic mucus gel. Biochem Soc Trans 1995;23:104S.

16 Van-Seuningen V, Davril M. A rapid periodic acid-Schiff staining procedure for the detection of glycoproteins using the PhastSystem. Electrophoresis 1992:13:97-9.

17 Hutton DA, Pearson JP, Allen A, et al. Mucolysis of the colonic mucus barrier by proteases: inhibition by interacting polyacrylate. Clin Sci 1990;78:265-71.

18 Pearson JP, Allen A, Venables CW. Gastric mucus: isolation and polymeric structure of the undegraded glycoprotein: its and polymeric structure of the undegraded glycoprotein: it
breakdown by pepsin. Gastroenterology 1980;78:709-15.

19 Mantle M, Allen A. A colorometric assay for glycoproteins based on the periodic acid/Schiffs stain. Biochem Soc Trans 1978;6:607-8

20 Younan F, Pearson JP, Allen A, et al. Changes in the structure of the mucus gel on the mucosal surface of the stomach in association with peptic ulcer disease. Gastroenterology 1982;82:827-31.

21 Kuipers EJ, Lee A, Klinkenberg-Knol EC, et al. Review article: the development of atrophic gastritis-Helicobacter pylori and the side effects of acid suppressive therapy. Aliment Pharmacol Ther 1995;9:331-40

22 Kerss S, Allen A, Garner A. A simple method for measuring the thickness of the mucus gel layer adherent to rat, frog, and human gastric mucosa: the influence of feeding, prostaglandin, $\mathrm{N}$-acetylcysteine and other agents. Clin Sci 1982;63:187-95.

23 Ota H, Katsuyama T. Alternated laminated array of two types of mucin in the human gastric surface mucus layer. Histochem $\mathcal{F} 1992 ; 24: 86-92$.

24 Schade C, Flemstrom G, Holm L. Hydrogen ion concentration in the mucus layer on top of acid stimulated and inhibited rat gastric mucosa. Gastroenterology 1994;107:180-8.

25 Hidaka E, Goto A, Matsuzawa K, et al. Gastric surface mucous gel layer and H pylori [abstract]. Gut 1997; 41(suppl 1):A29.

26 Allen A. Gastrointestinal mucus. In: Handbook of physiology. The gastrointestinal physiology. The salivary, gastric and hepatobilary secretions. Section 6. Vol 111. Bethesda: American Physiological Society, 1989:359-82. 